\title{
Conductive Channel for Energy Transmission
}

\author{
Victor Victorovich Apollonov \\ A. M. Prokhorov General Physics Institute, Russian Academy of Sciences, Moscow 119991, Russia
}

Received: June 11, 2014 / Accepted: July 08, 2014 / Published: September 30, 2015.

\begin{abstract}
Laser spark obtained by using a conical optics is much more appropriate to form conducting channels in atmosphere. Only two types of lasers are actively considered to be used in forming high-conductivity channels in atmosphere, controlled by laser spark: pulsed sub-microsecond gas and chemical lasers $\left(\mathrm{CO}_{2}\right.$, DF (deuterium fluoride)), short pulse solid-state and UV (ultraviolet) lasers. Main advantage of short pulse lasers is their ability in forming of super long ionized channels with a characteristic diameter of $\sim 100 \mathrm{~mm}$ in atmosphere along the beam propagation direction. At estimated electron densities below $1,016 \mathrm{~cm}^{-3}$ in these filaments and laser wavelengths in the range of 0.5-1.0 mm, the plasma barely absorbs laser radiation. In this case, the length of the track composed of many filaments is determined by the laser intensity and may reach many kilometers at a femtosecond pulse energy of $\sim 100 \mathrm{~mJ}$. However, these lasers could not be used to form high-conductivity long channels in atmosphere. The ohmic resistance of this type a conducting channels turned out to be very high, and the gas in the channels could not be strongly heated $(<1 \mathrm{~J})$. An electric breakdown controlled by radiation of femtosecond solid-state laser was implemented in only at a length of $3 \mathrm{~m}$ with a voltage of $2 \mathrm{MV}$ across the discharge gap $(670 \mathrm{kV} / \mathrm{m})$. Not so long ago scientific group from P.N. Lebedev physical institute has improved that result, the discharge gap $(-1 \mathrm{~m}$ ) had been broken under $\mathrm{KrF}$ laser irradiation when switching high-voltage (up to $390 \mathrm{kV} / \mathrm{m}$ ) electric discharge by 100 -ns UV pulses. Our previous result $-16 \mathrm{~m}$ long conducting channel controlled by a laser spark at the voltage $-3 \mathrm{MV}$ was obtained more than 20 years ago in Russia and Japan by using pulsed $\mathrm{CO}_{2}$ laser with energy $-0.5 \mathrm{~kJ}$. An average electric field strength was $<190 \mathrm{kV} / \mathrm{m}$. It is still too much for efficient applications.
\end{abstract}

Key words: Laser, exploding wire, conductive channel, laser spark, electric discharge, pulse-periodic laser, energy transmission.

\section{Introduction}

For many years, the attempts to create super long conductive channels were taken in order to study the upper atmosphere and to settle special tasks, related to the energy transmission. There upon the program of creation of "Impulsar" represents a great interest, as this program in a combination with high-voltage high repetition rate electrical source can be useful to solve the above mentioned problems. It looks like as a kind of "renaissance of Nikola Tesla ideas" for the days of high power lasers. The principle of conductive channel production can be shortly described as follows. The "Impulsar"-laser jet engine vehicle-propulsion take place under the influence of powerful high repetition rate pulse-periodic laser radiation. In the experiments,

Corresponding author: Victor Victorovich Apollonov, professor, research fields: high power lasers and interaction of laser radiation with matter. E-mails: vapollo@rambler.ru, vapollo@kapella.gpi.ru. the $\mathrm{CO}_{2}$-laser and solid state $\mathrm{Nd}$ YAG (neodymium-doped yttrium aluminium garnet laser) laser systems had been used. Active impulse appears thanks to air breakdown $(<30 \mathrm{~km})$ or to the breakdown of ablated material on the board ( $>30 \mathrm{~km})$, placed in the vicinity of the focusing mirror-acceptor of the breakdown waves. With each pulse of powerful laser, the device rises up, leaving a bright and dense trace of products with high degree of ionization and metallization by conductive nano-particles due to the ablation process. Conductive dust plasma properties investigation in our experiments had been produced on the basis of two very effective approaches: high power laser controlled ablation of different materials and by electrical explosion of wire. Experimental and theoretical results of conductive canal modeling will be presented. The estimations show that, with already experimentally demonstrated figures of specific thrust impulse, the lower layers of the ionosphere can be 
reached in several hundred seconds that is enough to keep the high level of channel conductivity and stability with the help of high repetition rate high voltage generator. At present, many laboratories still continue the search for an efficient laser-based lightning protection system [1-3] and for producing a controlled high-conductivity channel in the atmosphere [4-8], capable of conducting a short-circuit current [9] in a natural or artificial electrical circuit. In Ref. [7], demonstrated a 1.5 -fold increases in the length of the discharge gap broken under laser irradiation when switching extended $\left(\begin{array}{ll}\sim 1 & \mathrm{~m}\end{array}\right)$ high-voltage (up to $390 \mathrm{kV}$ ) electric discharges by 100-ns UV pulses of a KrF laser. Laser-based lightning protection systems, as is known, rely on the so-called long laser spark, which provides the conditions for connecting a thunderstorm cloud with a grounded metal rod, i.e., a classical lightning rod. Maximum lengths $(\sim 16 \mathrm{~m})$ of the laser-spark-controlled electric discharge at a voltage of $3,000 \mathrm{kV}$ were obtained in Russia and Japan [3] using a $0.5 \mathrm{~kJ}$ pulsed $\mathrm{CO}_{2}$ laser with spherical optics. Such control conductivity channels can be used in energy transmission, overvoltage protection systems, transport of charged particle beams, plasma antennas, etc. [8].

\section{Lasers for Producing Sparks}

It was shown in Refs. $[10,11]$ that, a laser spark produced using conical optics demonstrates much better characteristics from the point of view of formation of conductivity channels in the atmosphere. Currently, among the huge variety of different lasers, only two types are being actively studied to be used in the formation of laser-spark-controlled high conductivity channels in the atmosphere: submicrosecond pulsed gas and chemical lasers $\left(\mathrm{CO}_{2}\right.$, DF (deuterium fluoride)) and femtosecond solid-state lasers $[4,6,12]$.

The main advantage of femtosecond lasers is the ability of producing superlong ionized channels (so-called filaments) with a characteristic diameter of about $100 \mu \mathrm{m}$ in atmosphere along the laser beam propagation direction. With an estimated electron density of $10^{16} \mathrm{~cm}^{-3}$ in these filaments and the laser wavelength in the range of $0.5-1.0 \mu \mathrm{m}$, the plasma hardly absorbs the laser light. In this case, the length of the track consisting of many filaments is determined by the laser intensity and can reach many miles at a femtosecond pulse energy of $\sim 100 \mathrm{~mJ}$. However, these lasers could not be used to form long high-conductivity channels in atmosphere. The ohmic resistance of the thus formed conducting channels turned out to be very high, and the gas in the channels could not be strongly heated (the femtosecond laser energy is less than $\leq 1 \mathrm{~J}$ ). In particular, an EB (electric breakdown) controlled by radiation of a femtosecond solid-state laser was implemented in Refs. [5, 6] only at a length of $3 \mathrm{~m}$ (with a voltage of $2 \mathrm{MV}$ across the discharge gap).

As shown in Refs. [13, 14], to form a high-conductivity channel in the atmosphere, which could commute an artificial or natural discharge (lightning), the gas must be heated to a temperature from $6,000 \mathrm{~K}$ to $8,000 \mathrm{~K}$, when thermal ionization begins. This condition is crucial, because the high initial electron density in the atmosphere is a necessary but not sufficient condition for realizing high conductivity in a long atmospheric channel. As was mentioned in Ref. [13], it is important not only to produce but also to maintain the electron density in an atmospheric channel that is sufficient for the breakdown. During gas heating, thermal ionization becomes the main mechanism of electron "production" in the channel. Specifically, this is the main reason why the streamer mechanism cannot lead directly to an electric breakdown in the presence of high electron density (filaments) in a cold channel [13].

In the case of submicrosecond $\mathrm{CO}_{2}$ or DF lasers with a high energy per pulse (several hundreds of joules), long conducting laser-plasma channels can be formed with conical optics. In contrast to spherical optics, one can use methods of lens dynamic correction to increase aberrations and elongate the focal segment by passing 
to a conical phase front [11]. For example, an axicon transforms the phase front of a plane wave so that it becomes conical rather than spherical and converges to the $\mathrm{z}$ axis at an angle $\gamma$. For small angles $\left(\gamma<1^{\circ}\right)$, it is simply expressed in terms of the angle $\gamma$ at the axicon base and the refractive index $N$ of the axicon material: $\gamma$ $=(N-1) \alpha$. Each ring element of a conical wave front with a radius $R$ and width $\delta R$ is focused into an axial element of length $\delta z=\delta R / \gamma$ and the entire focal segment has the length $L=R / \operatorname{tg} \gamma=R / \gamma[11]$. This length can be made significant. For example, at $R=10 \mathrm{~cm}$ and $\gamma=0.5^{\circ}$, it amounts to $L \approx 11 \mathrm{~m}$. The cylindrical symmetry of focusing suggests that, the type of transverse intensity distribution is independent of the coordinate $\mathrm{z}$, a situation corresponding to a "diffractionless" wave beam. In reality, such beams also undergo diffraction, however, the diffraction energy loss from the central part of the beam is compensated for by the distributed lateral supply of radiation. Note that, the theoretical length of the focal segment formed by the axicon is independent of the laser beam energy and power and is determined by only the beam diameter. At $R=100 \mathrm{~cm}$ and $\gamma=0.5^{\circ}$, it amounts to $L \approx 110 \mathrm{~m}$. According to our estimates, when using $\mathrm{CO}_{2}$ lasers with an energy of $\sim 5 \mathrm{~kJ}$ per pulse, the length of these channels for wide laser beams and conical optics can be fairly large (much more than $100 \mathrm{~m})$.

When laser radiation is focused by an axicon in a real experiment, the longitudinal intensity distribution $I(\mathrm{z})$ along the focal length $L$ depends on $\mathrm{z}$, since the transverse intensity distribution $I(R)$ of the input beam is transformed by the axicon into the longitudinal distribution $I(\mathrm{z})$ of the Bessel beam. When the radiation intensity in the beam reaches the threshold, there occurs a breakdown in the medium and an extended plasma channel is formed along the focal length of the axicon [11]. Initially, the channel has a diameter of 20-100 $\mu \mathrm{m}$ and a length that is on order of the focal length $L$ of the axicon. If the breakdown is stable, the plasma channel, in contrast to the case of spherical optics, becomes continuous for few nanoseconds. The density of the plasma, depending on the parameters of the medium, the wavelength $\lambda$, and other experimental conditions, varies from $10^{17}$ $\mathrm{cm}^{-3}$ to $3 \mathrm{~cm}^{-3}$, the temperature reaches $30-40 \mathrm{eV}$, and specific conductivity is $10(\mathrm{ohm} \cdot \mathrm{m})^{-1}$. A few microseconds later the channel expands to $10 \mathrm{~mm}$. Accordingly, the temperature drops to $1 \mathrm{eV}$ and retains at this level for about $100 \mu \mathrm{s}$, followed by a relaxation of the plasma [11].

Fig. 1a shows a photograph of a plasma channel in scattered heating radiation with a wavelength $\lambda=1.06 \mu \mathrm{m}$

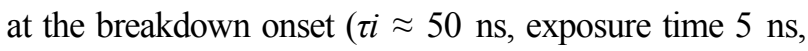
delay time $t_{\mathrm{d}}=0 \mathrm{~ns}$ ) [11]. The channel structure is affected by the combination of such parameters as the pulse width and the angle $\gamma$. For example, for a long pulse, laser-supported detonation may arise at inhomogeneities of the channel structure [15], as a result of which the structure becomes herringbone [11]. The photograph of a CLS (continuous laser spark) in Fig. $1 \mathrm{~b}$ was obtained by focusing $\mathrm{CO}_{2}$-laser radiation in the atmosphere by a conical mirror with an opening angle $\gamma=177.8^{\circ}$ [8]. One can see that, a laser spark looks like a continuous cord, composed of bright tapered elements. There are no discontinuities that are characteristic of sparks produced by focusing radiation by spherical optics. A similar pattern was observed at laser energies below $300 \mathrm{~J}$ [8]. In the photograph of a CLS fragment in Fig. 1c, the longitudinal structure of the spark looks like a set of bright tapered elements, which are in contact and equally spaced. Fig. 1d shows a photograph of the electric breakdown in the atmosphere for a channel formed by a CLS. When studying the spark between two rods immersed in its plasma and spaced by $d=80 \mathrm{~cm}$ [8], an electric breakdown occurred at a laser energy of $140 \mathrm{~J}$ and minimum average electric field strength $E_{\min } \approx 77 \mathrm{~V} / \mathrm{cm}$.

The up-to-date experiments aimed at implementing laser-spark-guided electric discharges shows another fundamental difference in the case of femtosecond solid-state and long-wavelength lasers. In the presence 


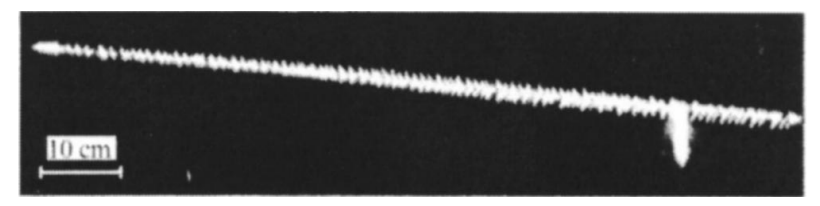

(a)

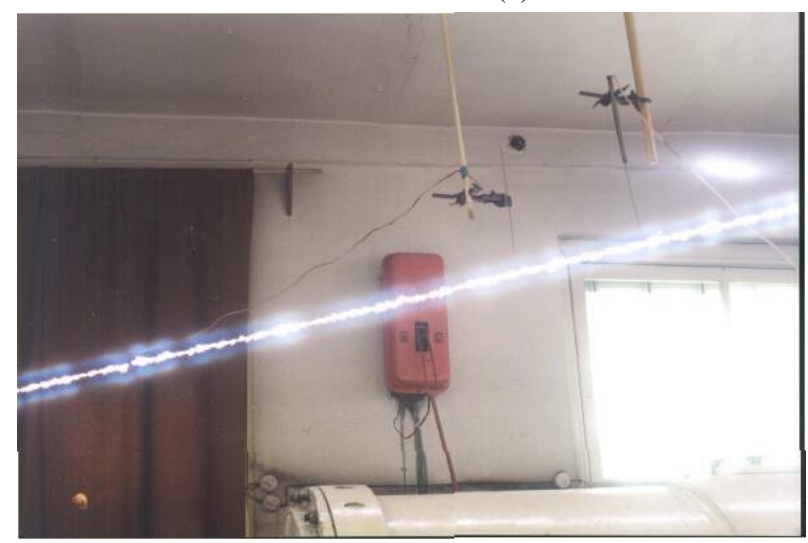

(c)

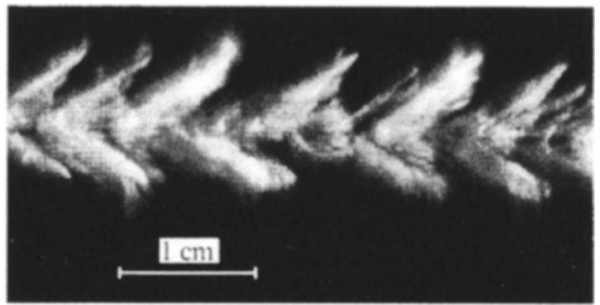

(b)

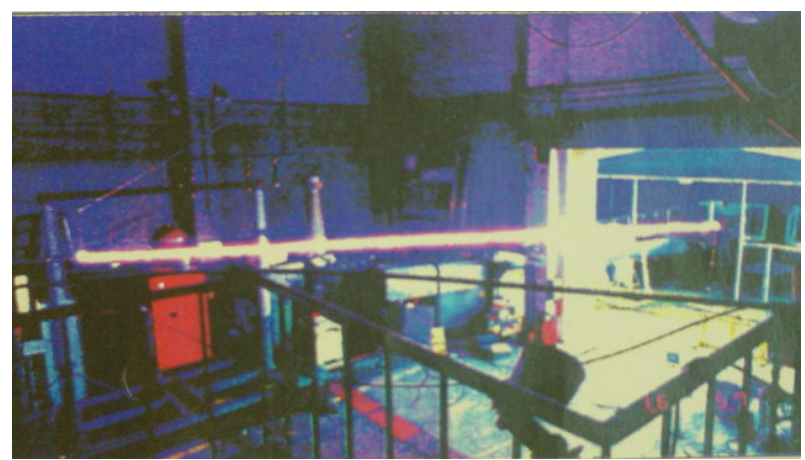

(d)

Fig. 1 (a) Schematics of focusing laser radiation by a CL (conical lens) and a photograph of the channel in scattered heating LR (laser radiation) with a wavelength $1.06 \mu \mathrm{m}$ at the breakdown onset (50 ns, exposure time $5 \mathrm{ns,}$ delay time $=0$ ); (b), (c) photographs of a continuous laser spark for $\mathrm{CO}_{2}$-laser radiation focused by a conical mirror with an opening angle $=177.8^{\circ}$; and (d) a photograph of electric breakdown in atmosphere in a CLS-induced channel.

of a laser-plasma channel composed of filaments, the breakdown voltage decreased by $30 \%$ [5]. The use of $\mathrm{CO}_{2}$ and DF lasers made it possible to reduce it by a factor of more than 10 .

As was pointed out in Ref. [16], the guiding properties of CLS (with a high voltage delayed by $10-20 \mu \mathrm{s}$ ) are similar to the action of a chain of metal balls. After an optimal time of 10-20 $\mu$ s, the expansion of individual breakdown "zones" leads to the formation of a continuous quasi-cylindrical conducting channel with a low gas density $(\sim 0.1$ of the normal density), high equilibrium temperature $(2,700-2,900 \mathrm{~K})$, and electron density in the range from $10^{7} \mathrm{~cm}^{-3}$ to $10^{8} \mathrm{~cm}^{-3}$.

\section{Pulse-Periodic Laser}

Another approach to the formation of a conducting channel relies on the application of a pulse-periodic laser. It was shown in Ref. [17] that, electric-discharge and gas-dynamic lasers with a high peak power can operate in a high-frequency pulse-periodic regime.
This lasing regime allows one to produce a long conducting channel in the atmosphere, which exists for a long time. To this end, it is necessary to align a laser beam with an inverted Cassegrain telescope system, which consists of two spherical mirrors. The first (main) mirror of large diameter is immobile, while the second mirror can move according to a certain law. When moving the second mirror, the common real focus of the system changes its position in time from infinity to some minimum value, which is determined by the optical scheme of the telescope. Calculations show that, at a certain repetition frequency of laser pulses and a certain motion law for the second mirror, continuous extended plasma channels can be formed. However, practical implementation of this scheme with a "running" focus meets a number of fundamental difficulties. The light distribution in the focal plane, yielded by a well correlated lens, is in essence due to the Fraunhofer diffraction. The specific features of the out-of-focus 3D monochromatic images of a point 
source, obtained with a round hole, were considered for the first time by Lommel. Along the optical $\mathrm{z}$ axis, the length of the focal segment that determines the longitudinal size of the energy-accumulation region depends quadratically on the focal length of the system. The transverse size of this region is proportional to the focal length and the angular divergence of laser radiation, therefore, the volume within which energy is concentrated is proportional to the focal length in the fourth power. For this reason, to form a homogeneous plasma channel several kilometers long, one must use a pulse-periodic laser with a shaped pulse energy and the second telescope mirror moving according to a complex program. A practical implementation of this method with a pulse-periodic $\mathrm{CO}_{2}$ laser with an average power of $1 \mathrm{~kW}$ yielded a continuous plasma channel $\sim 1$ m long [18].

\section{Formation of a Current-Conducting Channel}

A completely different approach to the formation of a current-conducting channel can be realized using laser firing according to the "Impulsar" program [19]. Motion of an "Impulsar" laser engine under irradiation by a high-frequency pulse-periodic laser with a pulse energy sufficient for producing a breakdown in the focus of the optical system of a LRE (laser reactive engine) gives rise to a continuous conducting channel in air as a result of the formation of a mixture of aerosol [20] with atmospheric air and its subsequent ionization [19]. In this case, according to the calculations, the aforementioned range of laser pulse repetition rates provides continuity of the conducting channel at optimal velocities of the focusing system. Indeed, each laser pulse focused by the optical system forms some extended plasma region with a high conductivity, which occupies a relatively small segment of the focusing system trajectory. If the laser pulses have a low repetition rate [21], these regions will form somewhat like a dashed line at some velocities of the focusing system. If the pulse repetition rate exceeds
$10 \mathrm{kHz}$ and the motion velocities are optimal for solving the problem stated, these high-conductivity regions will have no discontinuities, and a continuous conducting channel will be formed. However, when the moving optical system enters rarefied atmospheric layers, a problem of medium deficit arises. To solve it, the displaced optical system must have an efficient source of an appropriate medium (a material containing microscopic metal particles, which can easily be sublimated under laser irradiation) in the vicinity of the focus. In particular, one can use copper oxide $(\mathrm{CuO})$ nano-powder [22], which provides synthesis of conducting aerosol copper particles upon laser heating. The presence of aerosol particles of heavy metals in the sublimated material makes it possible to increase the specific impulse of the LRE thrust, simultaneously with increasing the channel conductivity.

To implement a long conducting channel, one must solve several problems:

- development of a high-frequency pulse-periodic $\mathrm{CO}_{2}$ laser with an average power $P \approx 100 \mathrm{~kW}$ and a peak power sufficient for initiating a breakdown in the focus of the LRE optical system;

- development of a system of dynamic correction for the wave front of a wide-aperture laser beam with a diameter $D$ \# $10 \mathrm{~cm}$ in the presence of small-scale turbulence in atmosphere;

- consideration of the laser radiation absorption and scattering by aerosol particles in the LRE exhaust;

- alignment of the starting position of LRE with the laser and the pulsed high-voltage (up to several MV) source;

- obtainment of maximally possible specific impulse of the LRE thrust to reduce the time of aerosol channel formation;

- choice of the material of aerosol heavy-metal particles and their dispersion composition that could provide high channel conductivity upon heating to the sublimation temperature in the LRE;

- determination of the lower limit for the size of dispersion aerosol particles to ignite electric 
breakdown of a channel capable of transmitting a current, corresponding to a short-circuit current in natural or artificial electric circuits at a minimally possible average strength of electric field.

To address the latter two problems, we conducted a series of experiments. The results obtained are reported below. We consider urgent the problems of determining the dynamics of change in the channel conductivity at long delay times, and its maintenance at the optimal level by introducing additional high-frequency generators into a chain, they will be analyzed in the next stage of the "Impulsar" program.

\section{Channel Formed by an Exploding Thin Wire}

The formation of a conducting channel based on the Impulsar technology was simulated using the results of the laboratory experiments on a controlled electric breakdown in an atmospheric channel containing aerosols [22, 23] of hot copper and copper oxide $(\mathrm{CuO}$, $\mathrm{Cu}_{2} \mathrm{O}$ ) particles, formed as a result of wire explosion. Note that, the proposed method for generating particles of variable size with a necessary concentration appears to be fairly efficient in terms of the price-quality criterion when one must carry out many experiments aimed at modeling the optimal conditions for high conductivity. There are a number of factors facilitating the electric breakdown (including the streamer stage) of long gaps in an atmospheric channel formed as a result of wire explosion at average external electric fields.

As noted in Ref. [14], the main problem in the analysis of the formation of a spark channel in a discharge gap in atmosphere using a field of arbitrary configuration is to determine the mechanism of gas heating, which provides thermal ionization of the gas in the spark channel. The heating in the discharge channel was generally believed to be caused by the increase in the concentration of the charged particles supplied by shock gas ionization. However, the concentration of charged particles in the range of $10^{11}-10^{13} \mathrm{~cm}^{-3}$ is limiting for shock ionization. The development rate of thermal processes in a gas is limited by the velocity of the electrons that transform the electric field energy to the thermal energy of gas molecules. The thermal inertia of the gas excludes the possibility of step changes in its temperature and therefore, its conductivity, both during heating and cooling the channel.

It was noted in Ref. [13] that, ionization in a highly ionized plasma differs significantly from the similar process in a weakly ionized plasma, where molecules are ionized by the electrons gaining energy directly from the electric field. A field in a highly ionized plasma supplies energy to all gas electrons. The latter are thermalized as a result of collisions and obey Maxwell's distribution. The gas is ionized by the electrons that acquire sufficient energy during the energy exchange with other particles rather than directly from the field. It is much easier to maintain a highly ionized equilibrium plasma than a weakly ionized nonequilibrium plasma, because the former situation requires a much weaker field. For example [13], in an equilibrium plasma column of nitrogen arc at atmospheric pressure, which burns in a cooled tube of radius $r=1.5 \mathrm{~cm}$ at a current $i=10 \mathrm{~A}$, the field supporting the arc column has a strength $E=10 \mathrm{~V} \cdot \mathrm{cm}^{-1}$. Under these conditions, $T=8,000 \mathrm{~K}$, the gas density $N_{\mathrm{a}}$ $=10^{18} \mathrm{~cm}^{-3}, n_{\mathrm{e}}=2 \times 10^{15} \mathrm{~cm}^{-3}$, the degree of ionization $x_{1}=n_{\mathrm{e}} / N=2 \times 10^{-3}$, and $E / N_{\mathrm{a}}=10^{-17} \mathrm{~V} \cdot \mathrm{cm}^{2}$. In a nonequilibrium glow discharge column in nitrogen, the strength of the discharge-supporting field is larger by an order of magnitude: $E=200 \mathrm{~V} \cdot \mathrm{cm}^{-1}$. Such a sharp difference is caused by the difference in the nature of ionization processes. In the case of weakly ionized plasma, the field must be sufficiently strong to accelerate electrons to an energy of $13-15 \mathrm{eV}$ (ionization threshold). At the same time, for highly ionized equilibrium plasma, the field can be much weaker, because in this case, it is sufficient to accelerate electrons to only $k T \approx 1 \mathrm{eV}$. As indicated in Ref. [13], electrons supply atoms with energy, and, 
during the energy exchange, all particles jointly concentrate energy in separate electrons, which serve as ionization sources.

Our experiments on the formation of a high-conductivity channel in atmosphere was performed with a PEV (personal electric vehicles)-2 copper wire in an enamel shell, the wire was 90-155 cm long and had a diameter of $90 \mathrm{~mm}$. Fig. 2a shows a schematic of the experimental setup, the arrangement of the sensors for measuring current by a Rogowski loop and voltage on the capacitor battery using a voltage divider, and the position of the end face of polished quartz fiber, which transfers radiation from the discharge channel to a high-speed pin-photodiode. The end face of the polished quartz fiber was located at a distance of $2-10 \mathrm{~cm}$ from the wire axis near the discharge cathode or anode. The shape of the current pulse was measured using a differential screened Rogowski loop with a time resolution of $\sim 30 \mathrm{~ns}$ [24]. The shape of the voltage pulse across the discharge gap was determined with the aid of a screened voltage divider of mixed type (connected to the capacitor battery anode), which

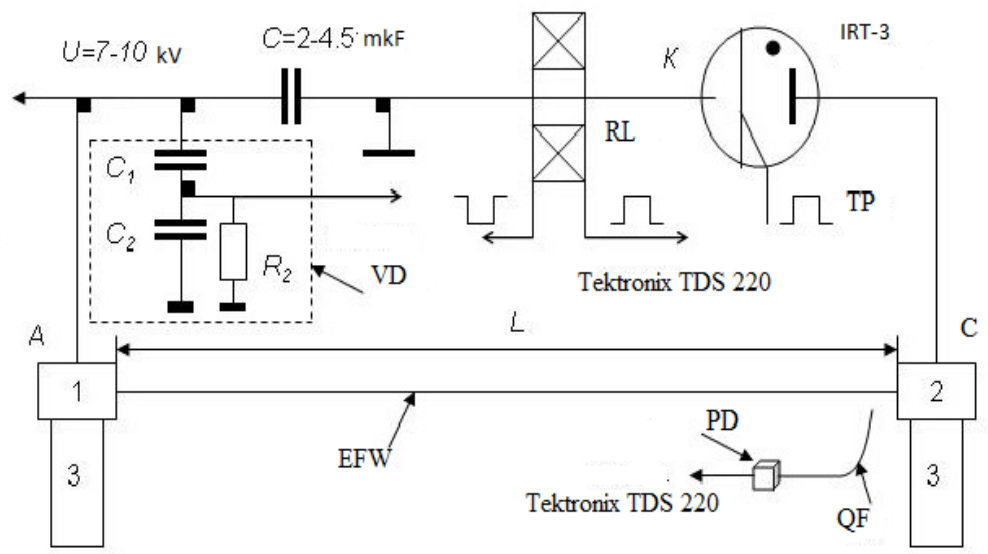

(a)

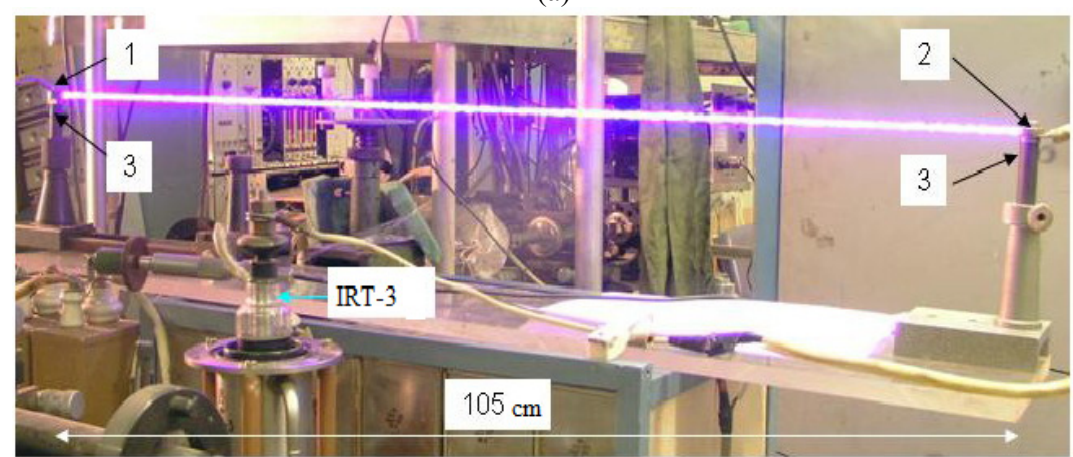

(b)

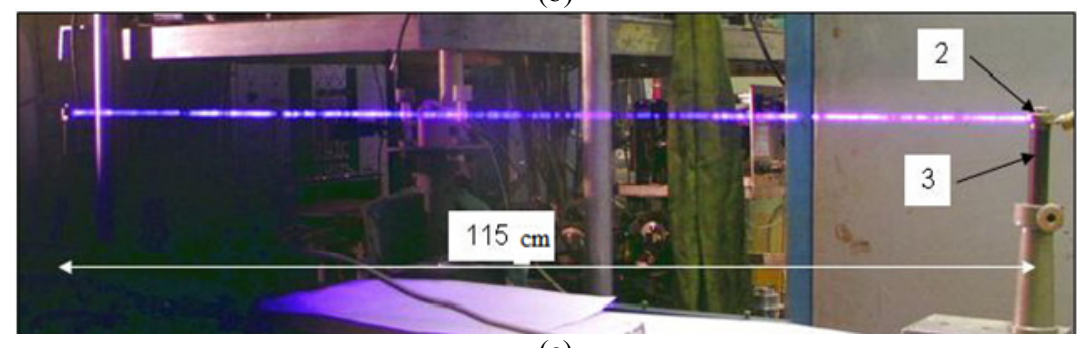

(c)

Fig. 2 (a) Schematics of the experiment on igniting an electric breakdown in a wire-explosion channel for a horizontally oriented wire: $(1,2)$ brass electrodes, (3) ebonite insulators, IRT-3 (ignitron switcher), RL (Rogowski loop), VD (voltage divider), TP (triggering pulse), EFW (exploding fine wire), PD (photodiode), QF (silica fiber), A (anode) and C (cathode); (b) a photograph of controlled electric breakdown; and (c) a photograph of wire explosion in the absence of breakdown. 
yields a measurement error of $\sim 1 \%$ at a width of the current-pulse leading edge $>1 \mu$ s [25]. Recording was performed using a Tektronix TDS 220 digital oscilloscope. The capacitor with a capacitance $C=2 \mu \mathrm{F}$ was charged from a voltage source to $U_{0}=7-10 \mathrm{kV}$. When switched on by an external triggering pulse from a controlled discharger (IRT-3 ignitron), the capacitor was discharged through a copper wire $90 \mu \mathrm{m}$ in diameter (with allowance for the enamel shell, the wire diameter was $\sim 110 \mu \mathrm{m})$. As the experiments showed, the presence of an enamel shell facilitates the formation of a thin straight-line electric breakdown channel with a conducting region 4-8 $\mathrm{mm}$ in diameter in atmosphere and promotes a unique mechanism of aerosol formation in the form of balls $330-20 \mu \mathrm{m}$ in diameter with a black surface (oxidized during wire explosion). Large pieces of the enamel shell (up to $10 \mathrm{~cm}$ long) remain integer, with burn-through areas on the surface. During the wire explosion and electric breakdown, the enamel shell stabilizes the spatial arrangement of the balls in the channel, which are centers of ionization plasma formations.

Fig. $2 b$ shows a photograph of electric breakdown in a channel formed during explosion of a horizontally oriented wire (D $90 \mu \mathrm{m}$, length $105 \mathrm{~cm}$ ). This photograph was recorded through an FS-1 blue filter and a neutral NS-9 filter by an OLYMPUS C-5050 ZOOM digital camera. At an exposure of $1.6 \mathrm{~s}$ the photographs have an integral character, i.e., the exposure during photographing the discharge was determined by the discharge duration. The digital camera matrix operated with a maximum resolution. When taking pictures of the entire discharge channel, the distance $l$ from the wire centre to the digital camera objective was $\sim 2 \mathrm{~m}$. To reveal the electric breakdown details, sizes of individual hot particles formed during the wire explosion and the diameter of the channel and the inhomogeneities in the structure of channel luminescence, we took pictures in the macroscopic regime, in which the digital camera objective was located rather close (at $25-50 \mathrm{~cm}$ ) to the wire. When taking pictures of the wire explosion products, $l$ was chosen to be $3 \mathrm{~cm}$ (super macroscopic regime). The digital camera was used in the ZOOM regime. The spherical shape of the wire explosion products $(D$ $30-20 \mu \mathrm{m})$ is primarily explained by the effect of surface tension forces on the molten copper drops during their formation. The large spread of particle diameters is explained by the fragmentation and aggregation of metal drops during wire explosion [26]. The black color of the particles is due to the formation of copper oxide on their surface as a result of the interaction of copper drops with air oxygen. The oxidation is reversible, i.e., upon heating to $\sim 1,200 \mathrm{~K}$, copper oxide is reduced to copper [22]. During the wire explosion, the oxygen concentration in the channel formed should sharply decrease as a result of the oxidation. It was shown in this experiment that, by the instant of electric breakdown, the voltage across the discharge gap $U_{\mathrm{a}}=7 \mathrm{kV}$ provides the electric field strength in the channel $E_{\mathrm{c}}=U_{\mathrm{a}} / d \approx 67 \mathrm{~V} \cdot \mathrm{cm}^{-1}$.

At the storage capacitance $C=2 \mu \mathrm{F}$, depending on the charging voltage and wire length, wire explosion may occur without electric breakdown. Fig. 2c shows a photograph of a channel (D $90 \mu \mathrm{m}$, length $115 \mathrm{~cm})$ formed as a result of explosion in the absence of electric breakdown. It can be seen that the channel structure is intermittent: it consists of individual isolated plasma zones of superheated drops of wire material [27-29]. At a charging voltage $U_{0}=10 \mathrm{kV}$ and storage capacitance $C=2 \mu \mathrm{F}$, the voltage across the capacitor battery decreases to $7.6 \mathrm{kV}$ by the end of the wire explosion. At a constant charging voltage $U_{0}$, the maximum pulse current during wire explosion is mainly determined by the initial wire resistance $R_{0}$ and amounts to $1.25 \mathrm{kA}$. When changing the length of $90 \mu \mathrm{m}$ copper wire within $90-155 \mathrm{~cm}$, the measured resistance ranged from $2.7 \mathrm{~W}$ to $4.2 \mathrm{~W}$. At $U_{0}=10 \mathrm{kV}$ and $C=$ 2-4 $\mu \mathrm{F}$, the maximum amplitude of the pulse current was in the range of 1-1.4 kA. After the wire explosion, a current $i \approx 10$ A flows for some time through the hot channel and then decays to zero. As a result, the 
residual voltage across the capacitor battery decreases to $7 \mathrm{kV}$. Despite the presence of residual current after the wire explosion, which heats additionally the channel, the electric breakdown does not occur at these parameters of the discharge circuit. The amount of energy $W$ spent on wire explosion, calculated from the current and voltage characteristics, was found to be $\sim 35 \mathrm{~J}$.

In the series of experiments performed on a horizontally oriented wire, the capacitance of the capacitor battery was taken to be constant: $C=2 \mu \mathrm{F}$, the only variable parameters were the wire length (90-155 cm) and charging voltage $(7-10 \mathrm{kV})$. It was found experimentally that, at a wire length of $100 \mathrm{~cm}$, the minimum charging voltage at which there is no electric breakdown in the gap does not exceed $7 \mathrm{kV}$, a value corresponding to the minimum accumulated energy: $W_{0}=49 \mathrm{~J}$.

In the next series of experiments on the electric breakdown in a channel, we considered a vertically oriented wire. This version is preferred, because it takes into account the possible influence of the vertical component of electric field at the Earth's surface on the electric breakdown in the channel formed by wire explosion.

Fig. 3a shows a photograph of electric breakdown in a channel formed by a vertically oriented wire $(D 90 \mu \mathrm{m}$, length $143 \mathrm{~cm}$ ) at the initial stored energy $W_{0}=190 \mathrm{~J}$, which corresponds to the charging voltage $U_{0}=10 \mathrm{kV}$ and the storage capacitance $C=3.8 \mu \mathrm{F}$. The electric breakdown can be seen in the oscillograms of discharge channel current, voltage and luminescence, which were recorded by a high-speed pin-photodiode with a time resolution of $\sim 1$ ns. The light arrived at the photosensitive area of pin photodiode through a silica fiber $\sim 2 \mathrm{~m}$ long, whose polished receiving end face was located near the discharge anode at a distance of $1.5-4 \mathrm{~cm}$ from the discharge channel. The radiation pulse width at half maximum was $\sim 30 \mu$ s and hardly differed from the corresponding current pulse width during electric breakdown (the light-pulse oscillogram in Fig. 3e shows only the arc phase of the breakdown). The oscillogram of the discharge current (Figs. 3b and 3d) indicates that, it is close to zero during the current pause [30,31], whose duration is $\Delta T \approx 140 \mu \mathrm{s}$. The results of our studies showed that the current during the pause amounted to 10-50 A (depending on the discharge-circuit parameters).

There are many theories explaining this phenomenon, they will be considered elsewhere. As shown in Ref. [31], the electric breakdown delay for a copper wire depends on the average electric field strength $E_{\mathrm{c}}$ in the channel at the instant when the arc phase of the breakdown begins. The voltage oscillogram demonstrates that, the breakdown occurred at a voltage of $\sim 8.4 \mathrm{kV}$. Using the current and voltage oscillograms, one can also determine the channel resistance during the electric breakdown at the instant corresponding to $I_{\max }=360 \mathrm{~A}$. In this case, $R_{\min }=17 \mathrm{~W}$. Here, in contrast to the data of Refs. [32, 33], the discharge-channel diameter is of little importance, because the discharge is slow, and the inductive component can be neglected when estimating the voltage drop across the discharge channel. In Fig. 3b, which illustrates the case of the electric breakdown in a channel formed by wire explosion at $C=3.8 \mu \mathrm{F}$ and inductance $L=2 \mu \mathrm{H}$, the wave resistance $\rho=(L / C)^{0.5} \approx$ $0.7 \mathrm{ohm}$. Judging by the volt-ampere characteristics of the discharge gap, $L \approx 143 \mathrm{~cm}$ in the case of the electric breakdown at $R p \approx 18 \mathrm{ohm}, R p>2 \rho(18>1.4 \mathrm{ohm})$, which corresponds to the aperiodic shape of the discharge.

It was noted in Ref. [9] that, the channel resistance is affected by the amount of energy released in the channel rather than the current through the latter. At a wire length of 143-152 $\mathrm{cm}$ and the maximum capacitance $C_{\max }=4.5 \mu \mathrm{F}$, a breakdown in a channel formed by wire explosion occurred with a probability close to $100 \%$. Fig. $3 \mathrm{f}$ shows a photograph of a controlled electric breakdown at $C=4.5 \mu \mathrm{F}, U_{0}=10 \mathrm{kV}$, and an interelectrode distance of $155 \mathrm{~cm}$. In this experiment, an electric breakdown was ignited at a 


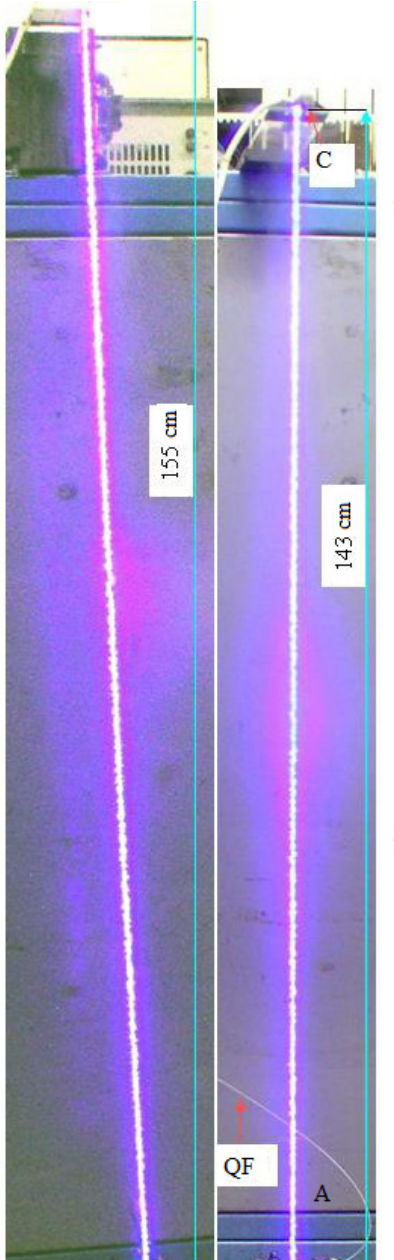

(f) (a)

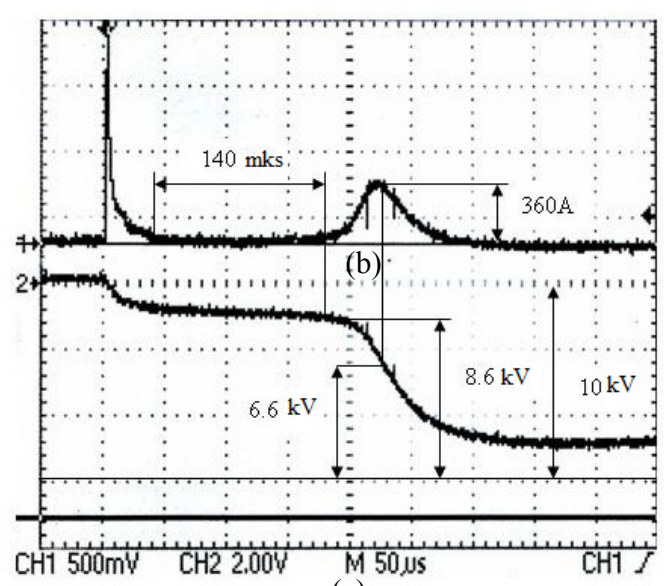

(c)

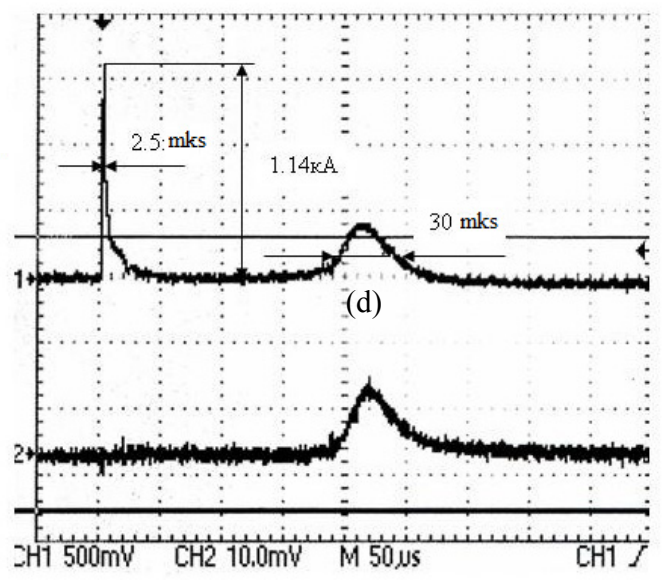

(e)

Fig. 3 (a) Photograph of controlled electric breakdown for explosion of a vertically oriented wire (the interelectrode length is $143 \mathrm{~cm}$ ); (b)-(e) oscillograms of the discharge channel ((b, d) current, (c) voltage, and (e) luminescence at a capacitance $C=3.8 \mu \mathrm{F}$ and charging voltage $U_{0}=10 \mathrm{kV}$ ); and (f) a photograph of controlled electric breakdown at $C=4.5 \mu \mathrm{F}$, charging voltage $U_{0}=$ $10 \mathrm{kV}$, and an interelectrode distance of $155 \mathrm{~cm}$.

voltage of $\sim 8.3 \mathrm{kV}$. The duration of the current pause was $\Delta T \approx 200 \mu \mathrm{s}$. At a wire length of $152-159 \mathrm{~cm}$, an electric breakdown occurred with a probability of $\sim 30 \%$. For wires longer than $159 \mathrm{~cm}$, the breakdown probability (at the same parameters of the scheme) was close to zero. Thus, the average minimum electric field strength in the channel formed by wire explosion, at which breakdown can occur, is $E_{\min } \approx 52 \mathrm{~V} / \mathrm{cm}$. The suggestion about the possibility of long-term support of current breakdown with an amount of accumulated energy increased by an order of magnitude and the same charging voltage was partially confirmed.

The channel for recording radiation in the visible wavelength range has a low sensitivity, because it detects only the radiation entering the angular aperture of the polished receiving end face of the quartz fiber. If the distance between the end face and the discharge channel is reduced to minimum $(\sim 1.5 \mathrm{~cm})$, one can detect the following phases in the oscillogram of a visible-light pulse. During the first $16 \mu$ s after the wire explosion, the visible light intensity is low (the wire explosion gives rise to radiation with wavelengths in the range of $\lambda \approx 2.5-0.5 \mu \mathrm{m}$ ) [29]. Then the oscillogram of visible light pulse exhibits the radiation corresponding to the streamer (weak), leader (weak), and arc phases of the breakdown. 
An analysis of many photographs indicates that, the electric breakdown trajectory (broken line, circle, etc.), which is set by spanned copper wire, is highly controllable and contains no branchings. The experiments revealed that the vertical orientation of exploded wire is indeed preferred in the case of streamer breakdown of maximally long gaps $\left(L_{\max } \approx\right.$ $159 \mathrm{~cm})$ at $U_{0}=10 \mathrm{kV}$ and $C_{\max }=4.5 \mu \mathrm{F}$.

Let us analyze the requirements to an ionized formation that can initiate electric breakdown. The minimal conditions that are necessary for initiating a streamer were reported in Ref. [13]. We will consider a uniform conducting sphere with a radius $R_{\mathrm{s}}$, placed in a uniform field of strength $E_{0}$. To form a self-propagating plasma channel, the external field must be displaced from the plasma formation and amplified beyond it. To displace the external field from the entire volume of the sphere, charges must be nonuniformly distributed over the surface, and there must be no less than $N=3 \varepsilon_{0} \pi R^{2} E_{0} / e$ electrons in the field. Taking into account that, the radius of this formation should be no less than $\alpha^{-1}$ ( $\alpha$ is the effective ionization coefficient), we can estimate the lower limit of the number of electrons at which a streamer may arise [13] as follows: $N e_{\min } \approx 3 \varepsilon_{0} \pi E_{0} / e \alpha^{2}$. As an example, we will consider air at atmospheric pressure. At the breakdown field strength $E_{0}=31.4 \mathrm{kV} / \mathrm{cm}$ and effective ionization coefficient $\alpha=12.4 \mathrm{~cm}^{-1}$, we have $N e_{\min }=2 \times 10^{9}$. The smaller the plasma formation radius, the more severe the limitation on the plasma density is. At the minimally allowable value $R \approx \alpha^{-1}$, the electron concentration in air at atmospheric pressure should be $N e_{\min } \approx 4 \times 10^{11} \mathrm{~cm}^{-3}$. The plasma formation size $R$ also must be no smaller than the ionization length $\alpha^{-1}$, in a field with a strength equal to the external field strength or even somewhat higher. In air at atmospheric pressure, the ionization length is $\alpha^{-1}$ $\approx 0.8 \mathrm{~mm}$. If the initial size of plasma formation in a homogeneous field satisfies the aforementioned requirements, this formation should generate ionization waves to both sides along the field, thus forming a plasma channel. The plasma channel development is accompanied by amplification of the field at both leader heads [13].

Our experiments showed that, all above requirements to an ionized formation [13], which must be fulfilled to ignite an electric breakdown, are implemented in the channel of the discharge gap formed by wire explosion with the aforementioned parameters. The characteristics of separate plasma formations ( $R_{\mathrm{s}} \approx 0.5-4 \mathrm{~mm}$, average distances $\sim 3-5 \mathrm{~mm}$, and temperature $T \approx 3,000-5,000{ }^{\circ} \mathrm{C}$ ) with clusters of superheated drops and their aggregates at the centre, as well as low oxygen content in the wire-explosion channel, reduce significantly the average field strength that is necessary for electric breakdown. Note that, the size of individual plasma formations exceeds that of superheated drops of wire material by one to two orders of magnitude. The average density of the wire-explosion products in the channel is $\rho \approx 0.1 \mathrm{~kg} / \mathrm{m}^{3}$. The electric field in separate spherical plasma fractions can be amplified by a factor of no more than 3 . Further studies should reveal new factors leading to electric breakdown in the channel produced by wire explosion at $E_{\mathrm{c}}=52 \mathrm{~V} / \mathrm{cm}[34,35]$. We should note the original work by Komel'kov [36], who analyzed the leader-discharge channel. We showed that, the specific resistance per unit length in the channel of leader positive retarded discharge is $11.8 \mathrm{~W} / \mathrm{cm}$, while the longitudinal gradient along the channel in the end of the leader phase is $55.5 \mathrm{~V} / \mathrm{cm}$. The main parameters of the leader channel turned out to be the same as in the case of arc discharge. The result obtained in Ref. [37] was interpreted in Ref. [13] as not quite correct because of incorrect analysis of the discharge-gap voltage oscillograms. Our results disprove the conclusion of Ref. [13].

To check the influence of the size of individual plasma formations in the channel on the mechanism of electric breakdown, we performed experiments with explosion of a copper wire $L \approx 30-36 \mathrm{~cm}$ long and $\varnothing 90 \mu \mathrm{m}$ in diameter at $U_{0}=10 \mathrm{kV}$ and $C_{\max }=4.5 \mu \mathrm{F}$, i.e., at a 
much higher specific energy contribution. The channel formed by wire explosion with these parameters contains a fairly large number of wire-material nanoparticles [22], whereas large particles (individual plasma formations) are completely absent. In this case, the second requirement to the development of streamer mechanism of breakdown in the channel is violated. As a consequence, a fivefold decrease in the gap length did not lead to breakdown at the corresponding parameters of the discharge circuit. It occurred only for wires not shorter than $L \geq 60 \mathrm{~cm}$, a minimum value at which the size of plasma formations in the wire explosion channel became sufficiently large to implement a streamer breakdown.

\section{Channel Produced by a Solid-State Laser}

We also investigated the regime of electric breakdown in a plasma channel containing evaporated aerosol particles of metals and their compounds, which is formed by focusing radiation of a solid-state laser, operating in the modified spike regime, onto targets made of different materials [38]. To form a plasma plume on a target, we used a phosphate glass laser with a wavelength $\lambda=1.054 \mu \mathrm{m}$, operating in a modified lasing regime with a peak power of $100-500 \mathrm{~kW}$. Photographs of lasers operating in this regime are shown in Figs. 4a and 4b. Solid-state lasers of this type are unique. Actually, they are laser hyperboloids (concerning the way of extracting radiation from a totally reflecting flat cavity through a round hole of large diameter, which can be seen in the upper photograph). Lasers of this type can produce large plasma plumes in both pulsed and pulse-periodic regimes. A laser pulse with a width up to $700 \mu \mathrm{s}$ consisted of many (approximately 150) high-contrast pulses 150-300 ns wide with an average repetition rate of $200 \mathrm{kHz}$ (Fig.4c). The radiation divergence at the laser output $\alpha \approx 4 \times 10^{-4} \mathrm{rad}$, was close to the diffraction one. The total energy per laser burst reached $\sim 30 \mathrm{~J}$ (see the laser in Fig. 4a). Graphite, vinyl plastic, textolite, ferrite, permalloy and carbonyl core were used as targets. Their exposure to focused laser radiation led to the formation of a cloud of rapidly expanding plasma, which contained aerosol particles of evaporated target material $\sim 50-1,000 \mathrm{~nm}$ in size. No large particles were found in the plasma cloud. Note that, a copper target cannot be used to form an electric breakdown in this plume, because evaporated large copper drops have velocities of several hundred meters per second and cannot form a quasi-stationary channel for electric breakdown. The conductivity of this plasma has been studied little, while the dynamics of changes in its properties is of great interest to search for the ways of forming (according to the Impulsar program) superlong high-conductivity channels in order to transfer energy at large distances. The experiments were performed with a system of electrodes, between which an electric discharge was ignited (Fig. 5). An anode in the form of a copper cylinder was connected to the high-voltage output of the capacitor battery with a capacitance $C=2 \mu \mathrm{F}$, which was charged to a voltage of $U_{0}=10 \mathrm{kV}$. A duraluminum cathode $(\mathrm{K})$ had a hole $\varnothing 6 \mathrm{~mm}$ in diameter, through which laser radiation was guided to the target using a lens with a focal length of $F$ $=300 \mathrm{~mm}$. The distance between the electrodes was $L$ $\approx 80 \mathrm{~mm}$. The photo of the plasma plume on the target made of graphite is shown in panel (Fig. 5b). The results of image processing on the PC with the use of the HSB (hue saturation brightness) color model are presented in panel (Fig. 5c).

At a laser pulse energy below $30 \mathrm{~J}$, the gap was switched using the plasma formed on the target. Typical photographs of the electric breakdown in atmosphere on a graphite target (at a laser burst energy of $12 \mathrm{~J}$ ) for a gap $\sim 70 \mathrm{~mm}$ wide, obtained using an FS-1 filter, a neutral NS-9 filter, and an OLYMPUS C-5050 ZOOM digital camera, are shown in Fig. 6. In highly inhomogeneous fields, streamers are formed primarily in the regions of maximum field strength near the electrodes and, depending on the voltage polarity, are directed either to the anode or to the cathode [13]. 


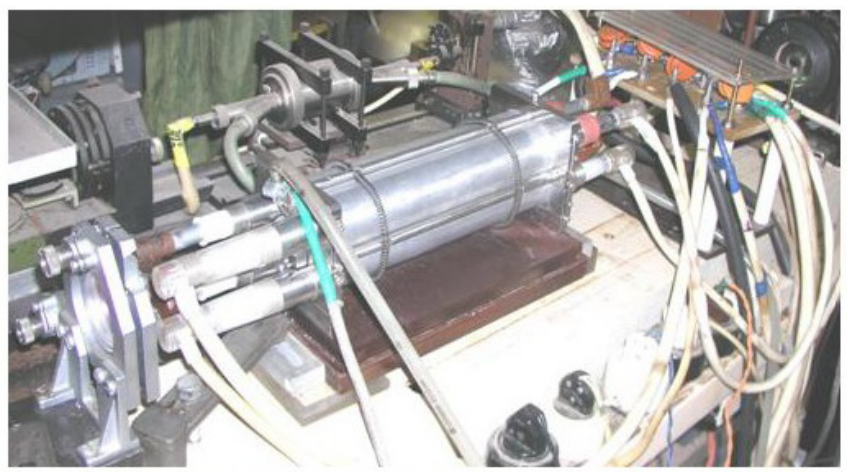

(a)

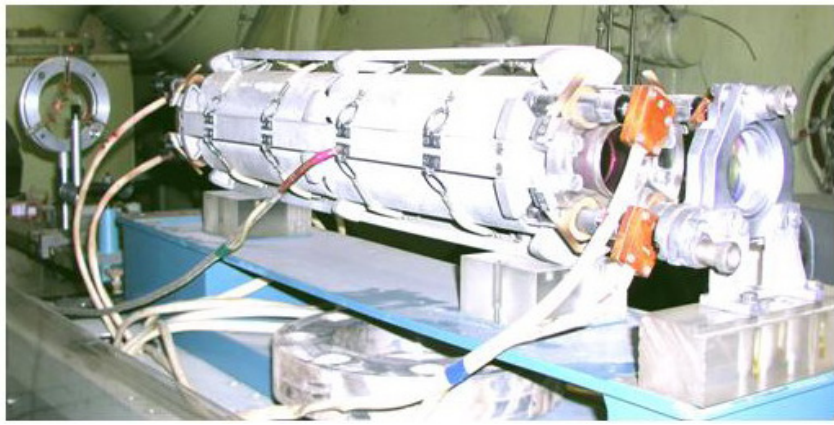

(b)
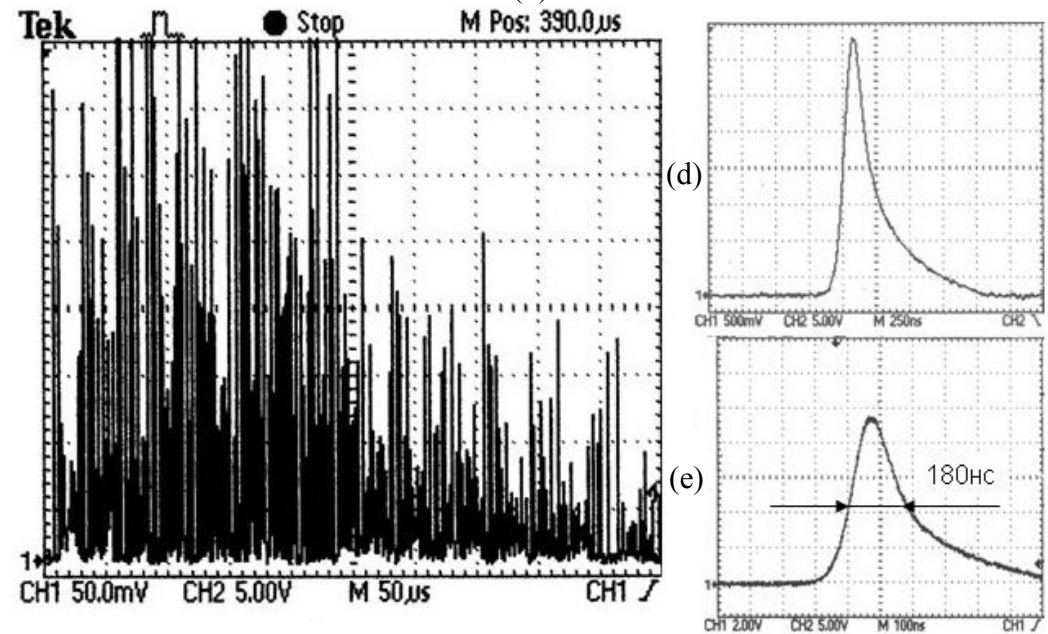

(c)

Fig. 4 (a), (b) General view of solid-state lasers; and (c)-(e) oscillograms of (c) the laser radiation; and (d), (e) separate laser pulses.

In the photographs in Fig. 6, the streamer directed to the anode develops more rapidly than that directed to the cathode. Streamers overlap at a distance of $\sim 25 \mathrm{~mm}$ from the anode, and an electric breakdown of the gap occurs. An analysis of this process shows that, the breakdown has a random trajectory, as well as a natural lightning. In addition, the results of experiments with different targets show that electric breakdown occurs at a minimum average electric field strength of $\sim 300 \mathrm{~V} / \mathrm{cm}$.
The experiments revealed also that, the electric breakdown delay (which is shown in the oscillograms of discharge-circuit current and luminescence) for the graphite target, could exceed $300 \mu$ s after the laser irradiation.

In Fig. 6c, the capacitance $C=2 \mu \mathrm{F}$. The current oscillogram indicates that, the damping oscillation period $T=8 \mu \mathrm{s}$, therefore, the discharge inductance is $0.8 \mu \mathrm{H}$. The contour wave resistance $\rho \approx 0.6 \mathrm{ohm}$. 
According to the current-voltage characteristics of the $L \approx 70 \mathrm{~mm}$ discharge gap for the graphite target, the discharge gap resistance is $R p \approx 0.5<2 \rho \mathrm{ohm}$, a value corresponding to an oscillatory discharge with a high damping decrement. The long breakdown delay with respect to the laser irradiation onset is apparently caused by the closing of the discharge gap by the expanding vapor of the target material and discharge-gap preionization by UV radiation of the plasma plume.

The targets made of other materials were characterized by a shorter possible delay. Changing the delay for the voltage pulse applied to the capacitor, one can measure the plasma characteristics at different instants and thus estimate the conductivity of the channel at different stages of its cooling and expansion. In our opinion, the strong electric field in these experiments is due to the absence of large particles in

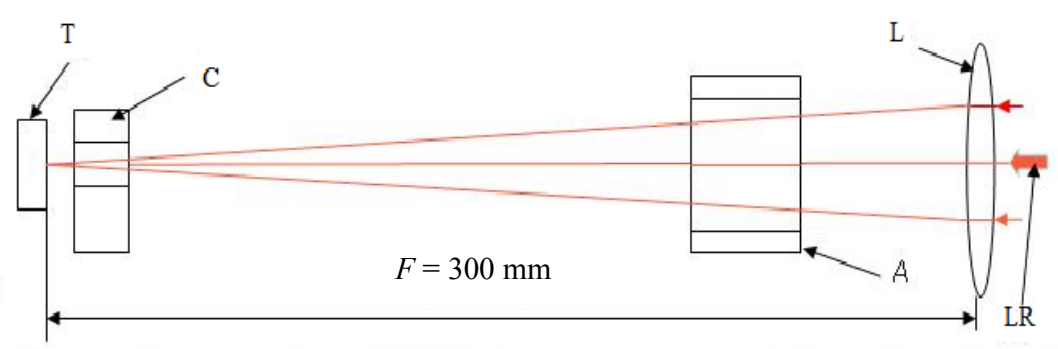

(a)

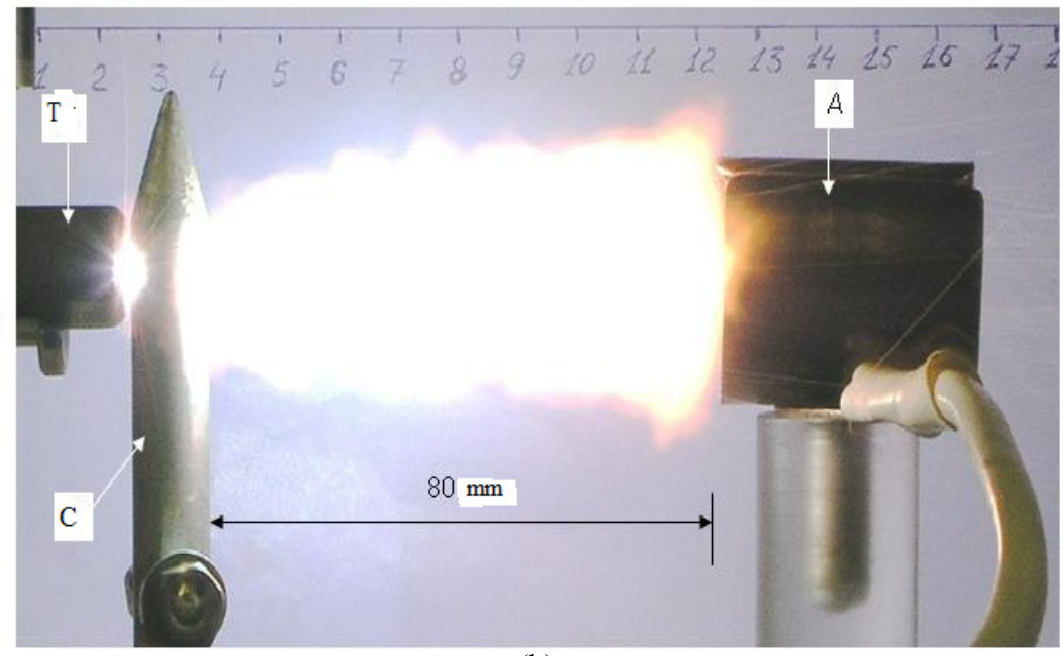

(b)

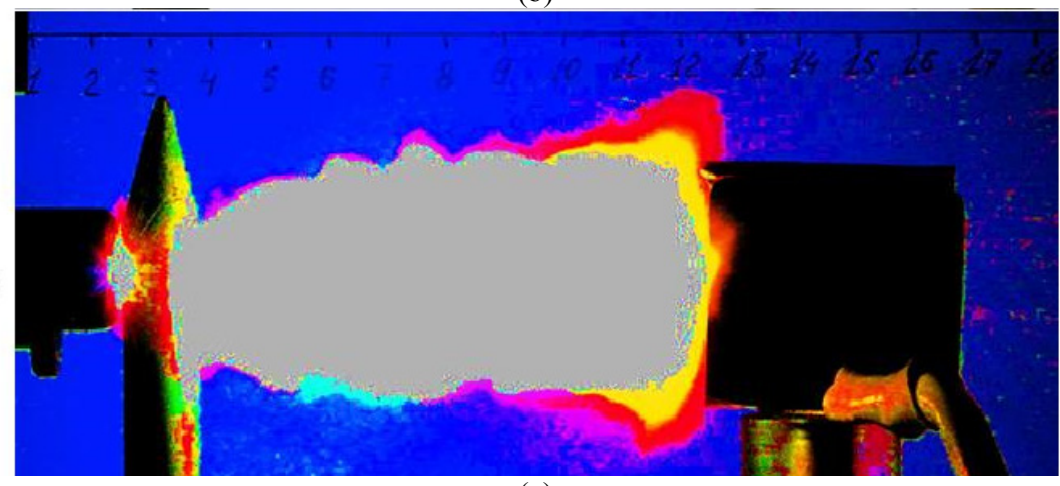

(c)

Fig. 5 (a) Schematics of the system for studying the conductivity of plasma plume products (T indicates a target); (b) a photograph of a plasma plume on a graphite target; and (c) the results of image processing in the system of HSB color coordinates. 


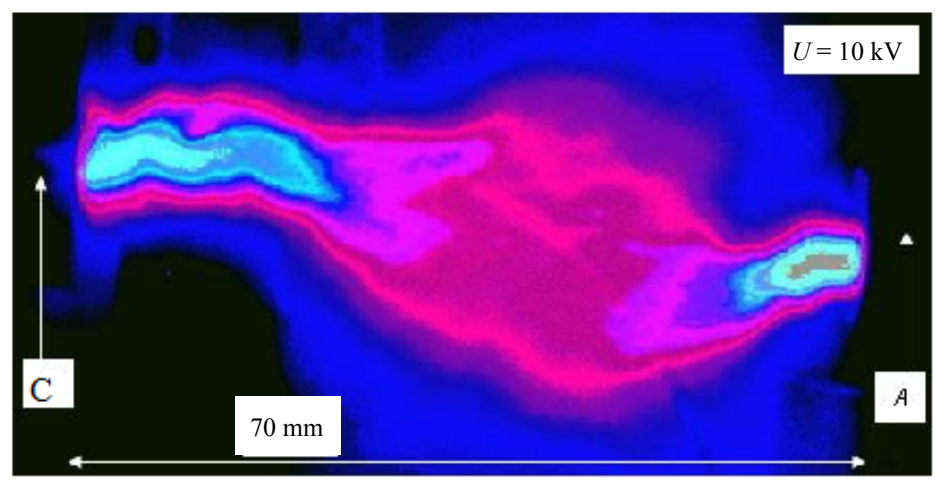

(a)

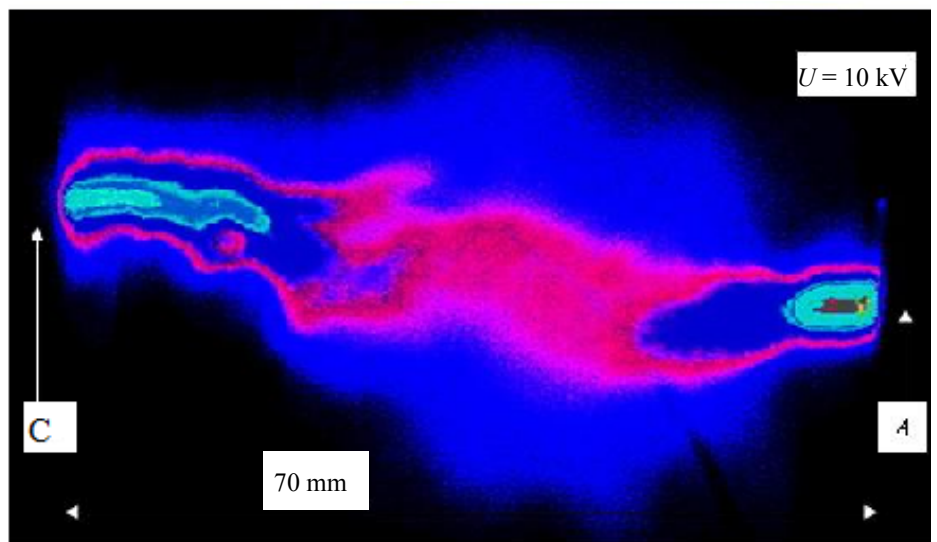

(b)

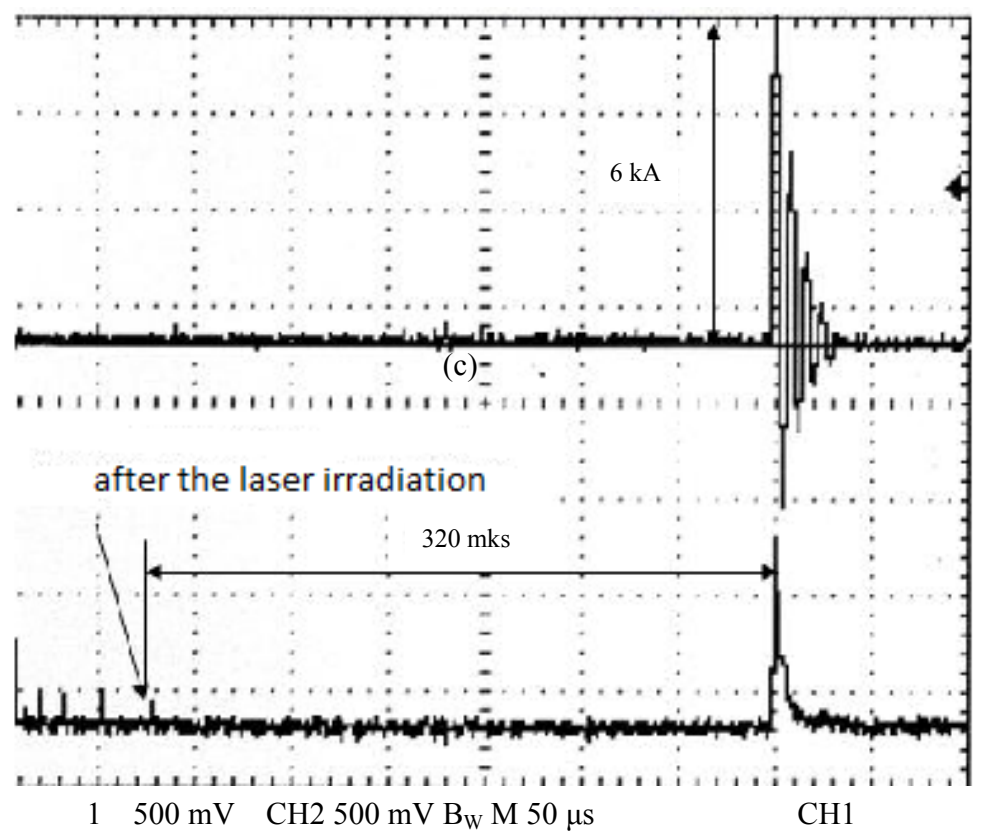

(d)

Fig. 6 (a), (b) Photographs of electric breakdown of a $70 \mathrm{~mm}$ gap in atmosphere in plasma-plume products (photographing through an FS-1 filter and a neutral NS-9 light filter) and the results of image processing in the system of HSB color coordinates; (c) a current oscillogram at electric breakdown of the gap (voltage $U_{0}=10 \mathrm{kV}$, storage capacitance $C=2 \mu \mathrm{F}$ ) in plasma plume; and (d) a luminescence oscillogram for the discharge channel in aerosol plasma. 
the erosion-plasma cloud. To carry out successfully the Impulsar program, we believe it necessary to study the dynamics of plasma conductivity and the residual aerosol products after cooling the plasma.

\section{Experimental Results}

Below we report the estimated values of the channel conductivity, which were obtained both in our previous experiments on the breakdown of atmospheric air using long- and short-wavelength radiation and the experiments with aerosol plasma (this study).

For the plasma channels obtained on graphite, ferrite, permalloy and carbonyl core targets using a phosphate glass laser, the gap specific resistance was $10 \mathrm{ohm} / \mathrm{m}$ and the specific breakdown voltage was $\sim 300 \mathrm{~V} / \mathrm{cm}$.

For the plasma containing a set of dispersion and condensation aerosols, which was obtained by wire explosion, the gap specific resistance was $\sim 5 \mathrm{ohm} / \mathrm{m}$ and the specific breakdown voltage was $\sim 50 \mathrm{~V} / \mathrm{cm}$.

For the plasma channel formed by a pulsed $\mathrm{CO}_{2}$ laser with a conical focusing optics, the gap specific resistance was $100-400 \mathrm{ohm} / \mathrm{cm}$ and the specific breakdown voltage was $\sim 80 \mathrm{~V} / \mathrm{cm}$.

For the plasma channel obtained with the aid of a femtosecond pulsed laser, the gap specific resistance was $\sim 0.1 \mathrm{MW} / \mathrm{m}$ and the specific breakdown voltage was $\sim 7.5 \mathrm{kV} / \mathrm{cm}[4]$.

These estimates, based on the results of the electric-breakdown experiments, show that a voltage of $7 \mathrm{MB}$, at a total introduced energy of $\sim 300 \mathrm{~kJ}$, is sufficient to form a plasma channel $\sim 1 \mathrm{~km}$ long and $8 \mathrm{~mm}$ in diameter [39, 40].

Note that, the minimum specific resistance of the channel depends strongly on the specific introduced energy, the transverse size of plasma formations, the concentration and size of aerosol particles, the repetition rate of laser pulses and their energy, and the humidity, temperature, and many other parameters of the medium where a conducting channel is formed.

The results of the studies show that, the discharge plasma has a high conductivity and can efficiently be used to form a conducting channel in order to transfer energy over long distances.

\section{Conclusions}

We determined the range of sizes of copper and copper oxide particles (from $50 \mathrm{~nm}$ to $300 \mu \mathrm{m}$ ) and their minimum volume density $\left(\sim 0.1 \mathrm{~kg} / \mathrm{m}^{3}\right)$ that are necessary to provide an electric breakdown in order to form a plasma channel according to the "Impulsar" program. The results of the model experiments showed that, nanoparticles in the plasma channel can merge into larger aggregates during the LRE operation.

The experiments on electric breakdown showed also that a high conductivity of a plasma channel with a diameter of $\sim 8 \mathrm{~mm}$ and a minimum specific resistance of $\sim 5 \mathrm{ohm} / \mathrm{m}$ is implemented at the maximum introduced specific energy: $\sim 200 \mathrm{~J} / \mathrm{m}$. At a larger channel diameter, the specific energy is proportional to the squared ratio of diameters.

The minimum average electric field strength $(\sim 52$ $\mathrm{V} / \mathrm{cm}$ ) in the channel, at which a streamer-leader electric breakdown of the discharge gap begins, is due to the following factors:

- the presence of dispersion and condensation aerosols of hot particles of copper oxide $\left(\mathrm{CuO}, \mathrm{Cu}_{2} \mathrm{O}\right)$ and copper with oxidized surface (smaller than $300 \mu \mathrm{m}$ in diameter) in the channel for a long time (1-10 $\mu \mathrm{s})$ due to their low mobility;

- the presence of superheated metal drops at the centre of plasma formations, which are in the metastable (unstable) state at temperatures $T \approx$ $3,000-5,000{ }^{\circ} \mathrm{C}$ and play a role of hot dots [31] during thermionic emission;

- formation of large linear aggregates of aerosol particles [20], which are formed during wire explosion;

- low oxygen content in the channel as a result of fast oxidation of aerosol particles formed during wire explosion.

\section{References}

[1] Apollonov, V. V. 1991. "High-Power $\mathrm{CO}_{2}$ and $\mathrm{N}_{2} \mathrm{O}$ Lasers with Volume Self-Sustained Discharge Pumping." 
J. Opt. Soc. Am. B 8 (2): 220.

[2] Alexandrov, G. N. 1980. Elektrichestvo 12 (2): 47.

[3] Kinoshita, F., Morooka, Y., and Uchiumi, M. 1997. Proceedings of the XII Intern. Conf. on Gas Discharge and Their Applications, 475.

[4] Diels, J. C., and Rudolph, W. 2006. Ultrashort Laser Pulse Phenomena: Fundamentals, Techniques, and Applications on Femtosecond Time Scale. Burlington: Acad. Press.

[5] Kasparian, J., Rodriquez, M., and M'ejean, G. 2003. "White-Light Filaments for Atmospheric Analysis." Science 301 (5629): 61-4.

[6] Berge, L., Skupin, S., Nuter, R., and Wolf, J. P. 2007. "Ultrashort Filaments of Light in Weakly Ionized, Optically Transparent Media.” Rep. Prog. Phys. 70 (10): 1633.

[7] Zvorykin, V. D., Levchenko, A. O., and Ustinovskii, N. N. 2011. "Control of Extended High-Voltage Electric Discharges in Atmospheric Air by UV KrF-Laser Radiation." Quantum Electron 41 (3): 227.

[8] Apollonov, V. V., Vasilyak, L. M., and Kazantsev, S. Y. 2002. "Electric-Discharge Guiding by a Continuous Spark by Focusing $\mathrm{CO}_{2}$-Laser Radiation with a Conic Mirror." Quantum Electron 32 (2): 115.

[9] Bazelyan, E. M., and Raizer, Y. P. 2001. Physics of Lightning and Lightning Protection. Moscow: Fizmatlit.

[10] Apollonov, V. V. 2005. "Feasibility Study of a $\mathrm{CO}_{2}$ Laser Based Lightning-Protection System Realization." Opt. Eng. 44 (1): 014302.

[11] Pyatnitskii, L. N., and Korobkin, V. V. 2000. Trudy IOFAN 57: 59.

[12] Teramobile. 2011. "Publications of the Teramobile Project." Teramobile. Accessed June 11, 2014. http://www.teramobile.org/publis.html.

[13] Bazelyan, E. M., and Raizer, Y. P. 1997. Spark Discharge. Moscow: Izd-vo $\mu$ FTI.

[14] Aleksandrov, G. N. 1967. Zh. Tekh. Fiz. 37: 288.

[15] Raizer, Y. P. 1974. Laser Spark and Propagation of Discharges. Moscow: Nauka.

[16] Asinovskii, E. I., and Vasilyak, L. M. 2000. Encyclopedia of Low-Temperature Plasma: Introduction. Book II. Moscow: Nauka, 234.

[17] Tret'yakov, P. K., Grachev, G. N., Ivanchenko, A. I., Krainev, V. L., Ponomarenko, A. G., and Tishchenko, V. N. 1994. Dokl. Akad. Nauk. 336 (4): 466.

[18] Apollonov, V. V., Kiiko, V. V., Kislov, V. I., Suzdal'tsev, A. G., and Egorov, A. B. 2003. "High-Frequency Repetitively Pulsed Operating Regime in High-Power Wide-Aperture Lasers." Quantum Electron 33 (9): 753.

[19] Grachev, G. N., Ponomarenko, A. G., Smirnov, A. L., Statsenko, P. A., and Tishchenko, V. N. 2005. "A Pulsating Optical Discharge Moving in a Gas."Kvantovaya Elektron 35 (11): 973.
[20] Apollonov, V. V. 2009. Proceedings of the X Intern. Conf. on Photonics and Optoelectronics, 13.

[21] Apollonov, V. V. 2009. Method for the formation of conducting channels in a nonconducting medium. RF Patent 2,400,005, filed May 20, 2009.

[22] Fuks, N. A. 1955. Mechanics of Aerosols. Moscow: Izd-vo AN SSSR.

[23] Ageev, V. P., Barchukov, A. I., and Bunkin, F. V. 1977. "Laser Air-Breathing Jet Engine." Sov. J. Quantum Electron 7 (12): 1430.

[24] http://www.nanosized-powders.com.

[25] Rukhadze, A. A., and Shpigel', N. S. 1965. Electric Explosion of Conductors. Moscow: Mir, 341.

[26] Gerasimenko, N. I., Grashina, N. A., Medvedkov, A. G., Meshcheryakov, A. B., and Pletnev, N. V. 1988. Prib. Tekh. Eksp. 1: 212.

[27] Goncharenko, G. M. 1963. "Electric Power Engineering." In MEI. Moscow: Izd-vo MEI, 146.

[28] Gavrilov, V. N., and Litvinov, E. A. 1993. Zh. Prikl. Mekh. Teor. Fiz. 34: 28.

[29] Kvartskhava, I. F., Plyutto, A. A., Chernov, A. A., and Bondarenko, V. V. 1956. Zh. Eksp. Teor. Fiz. 30: 42.

[30] Martynyuk, M. M. 1974. "Transport of Metal in Cathode Plasma Cluster at Explosion Emission of Electrons from Metallic Edges. II. Multiple Switching of Current." $Z h$. Tekh. Fiz. 44 (6): 1262.

[31] Peregud, B. P., and Abramova, K. B. 1964. Dokl. Akad. Nauk SSSR 157: 837.

[32] Abramova, K. B., Valitskii, V. P., Vandakurov, Y. V., Zlatin, N. A., and Peregud, B. P. 1966. Dokl. Akad. Nauk SSSR 167 (4): 778.

[33] Protopopov, N. A., and Kul'gavchuk, V. M. 1961. Zh. Tekh. Fiz. 31: 557.

[34] Aleksandrov, A. F., Zosimov, V. V., Kurdyumov, S. P., Popov, Y. P., Rukhadze, A. A., and Timofeev, I. B. 1971. "Dynamics and Radiation of Direct High-Current Discharges in the Atmosphere." Zh. Eksp. Teor. Fiz. 61 (November): 1841.

[35] Aleksandrov, A. F., and Rukhadze, A. A. 1976. Physics of High-Current Electric-Discharge Light Sources. Moscow: Atomizdat.

[36] Komel'kov, V. S. 1947. Dokl. Akad. Nauk SSSR 58: 57.

[37] Vlasto's, A. E. 1967. “Current Pause in Exploding-Wire Discharges.” J. Appl. Phys. 38 (13): 4993.

[38] Vlasto's A. E. 1968. "Restrike Mechanisms of Exploding Wire Discharges.” J. Appl. Phys. 39 (7): 3081.

[39] Apollonov, V.V., and Pletnev, N. V. 2004. Method for the inhomogeneous extraction of the energy of free higher transverse lasing modes from a laser and a laser. RF Patent 2,239,921, filed November 10, 2004.

[40] Apollonov, V.V. 2014. "High Power P-P Lasers." NOVA, N-Y. 